\title{
FUZZY APPROACH TO THE CAPACITATED FACILITY LOCATION PROBLEM
}

\author{
Lýdia GÁBRIŠOVÁ \\ Department of Mathematical Methods, Faculty of Management and Informatics, \\ University of Žilina, Univerzitná 8215/1, 01026 Žilina, Slovak Republic, \\ tel.: +421 41513 4285, e-mail: lydia.gabrisova@fri.uniza.sk
}

\begin{abstract}
The main objective of the paper is to devise and implement the algorithmic solution of problem of optimal location of set of facilities so that all demands of customers will be satisfied and the relevant costs of creating and maintenance of facilities are minimal. This optimization task can be modelled by capacitated facility location problem, which is the special class of integer linear programming problems. The output parameters of solution of such problems are the number of facilities actually used, the location of facilities and the assignment of individual customers to the concrete facility from the optimal set of facilities.

When a distribution system is to be designed, limits on terminal capability often must be taken into account. The capacity constraints in this case and also in other problems dealing with facility locations cause the severe difficulties in exact solving procedures because the underlying mathematical models are NP-hard. One possible approach is approximate method based on Lagrangean relaxation of the capacity constraints, which has several advantageous properties.

In capacitated location problems, the capacity of a facility as an upper limit of its ability to satisfy a given volume of demands cannot be precisely determined in most of practical applications. This circumstance evokes an idea to employ fuzzy approach for handling of the capacities and to utilize suggests an idea the fuzzy description in capacity constraint relaxation. The relaxed problem is exactly solvable even for real-world instances. It has been used in the heuristic method exploring the concept of measure of infeasibility.
\end{abstract}

Keywords: capacitated location problem, facilities, demands of customers, crisp problem, feasible solution, vagueness conditions, fuzzy approach, membership function

\section{INDRODUCTION}

Cost optimal design of the most of distribution and servicing systems consists in decisions on number and locations of facilities, from which demands of customers are satisfied. Such public or private servicing system is designed when some limits connected with the particular facilities emerge [4]. These constraints follow from the limited ability of the facilities to satisfy demands of customers. In spite of the fact that capacity of a facility is very vague value, the capacities of the located facilities bring serious complications concerning solving technique, which may be used to solve real-sized facility location problems.

In contrast to uncapacitated facility location problem, which can be solved exactly for real-sized instances containing hundreds of possible locations and thousands of customers, the capacitated location problem resists to all attempts to solve it exactly in a reasonable time. To avoid this complication and to obtain good solution of the capacitated facility location problem in a sensible time, we employ a fuzzy approach to handle the capacity limits and utilize an algorithm based on Erlenkotter's approach [1]. This approach relaxes the capacity constraints making use of a penalty function derived from membership functions describing customer clusters for individual facility locations.

The aim of this article is to design the distribution system in vagueness conditions, when the input capacities of the facilities are vague. When solving this task we will use fuzzy logic with the theorem that the task can be solved for deterministically stated data.

\section{SOLVING METHOD}

\subsection{Mathematical model of problem}

The capacitated facility location problem can be modelled mathematically as follows:

$$
\begin{array}{lll}
\text { Minimize } & \sum_{i \in I} f_{i} y_{i}+\sum_{i \in I} \sum_{j \in J} c_{i j} z_{i j} \\
\text { Subject to } & \sum_{i \in I} z_{i j}=1 \quad \text { for } j \in J \\
& z_{i j} \leq y_{i} \quad \text { for } i \in I, j \in J \\
& \sum_{j \in J} b_{j} z_{i j} \leq a_{i} y_{i} \quad & \text { for } i \in I \\
& y_{i}, z_{i j} \in\{0,1\} \quad \text { for } i \in I, j \in J
\end{array}
$$

Mathematical programming approach to the capacitated facility location problem assumes that goods distribution is performed from a primary source via warehouses to the particular customers. The number and positions of the warehouses, which will be called facilities in this paper, should be determined so that the total yearly cost for both located facilities and customer demand satisfaction is minimal.

The problem is described by a finite set of possible facility locations and a finite set of the customers, demands of which should be satisfied. Following costs and charges are connected to particular elements of these sets and to their relations.

A fixed charge for location of a facility at possible location $i$ is denoted by $f_{i}$. This fixed charge includes all 
costs connected with keeping this facility at the location for a given planning period, say one year. This charge does not include items, size of which depends on amount of demands, which are satisfied via this location.

The cost of $j$-th customer yearly demand satisfaction via facility located at place $i$ is denoted by coefficient $c_{i j}$. This coefficient includes all transportation costs for goods transport from the primary source to the facility location, from this location to the customer and manipulating cost in the facility.

It is presumed that a facility may be placed only at some place from finite set $I$ of possible locations. To model the decision on placing or not placing a facility at location $i$, variable $y_{i} \in\{0,1\}$ is introduced for each location $i$ from set $I$.

Let us denote by $J$ the set of all customers where each customer $j$ should be supplied by yearly amount of goods $b_{j}$. To be able to express that a customer belong to a given facility location and that he is supplied via this location, another set of zero-one variables is established. Variable $z_{i j}$ models a decision on assigning or not assigning customer $j$ to facility location $i$.

Let us consider that $a_{i}$ denotes the capacity of a facility located at $i$, then the complete model of the cost minimal capacitated facility location problem can be formed as (1) $-(5)$.

In this model, constraints (2) ensure that each customer demand is satisfied from exactly one facility location and constraints (3) enforce the placement of a facility at location $i$ whenever a customer is assigned to this facility location. Constraints (4) ensure that the total demand satisfied via facility location $i$ does not exceed given capacity $a_{i}, i \in I$.

\subsection{Approach with exceeding of given capacities}

Having omitted or relaxed constraints (4), the resulting problem (1)-(3), (5) is known as the uncapacitated facility location problem [2] and it can be effectively solved making use of implementation of the branch and bound method with Erlenkotter's lower bounding [1]. In our computation we use the BBDual algorithm for which it was shown that this approach is able to manage large size problems of practice [7], [8]. The large practical problem (1) - (5) integrality property of variables $y_{i}$ and $z_{i j}$ for $i \in I, j \in J$ and so it constitutes very hard problem for exact solving, when real-sized instance of problem is considered.

At this point we will sketch a possible approach to solving capacitated location problem:

By a simple relaxation or omitting the constraints (4) it is possible to simplify the model of capacitated location problem (1) - (5) to an ordinary uncapacitated location problem. Using the algorithm BBDual we get a solution, whose value of the objective function gives the first rough calculation of a lower bound of a solution of problem. By increasing the lower bound it is possible to search the unknown value of an objective function of the optimal solution of problem (1) - (5), which affects the quality of the final solution.

One of options of getting the lower bound is Lagrangean relaxation of the constraints (4). Its principle is to introduce nonnegative multipliers $u_{i}, i \in I$ into the objective function and transforming the problem into uncapacitated location problem with different objective function [9] change as stated below:

$$
\begin{aligned}
& \text { Minimize } \\
& \begin{array}{l}
\sum_{i \in I}\left(f_{i}-a_{i} u_{i}\right) y_{i}+\sum_{i \in I} \sum_{j \in J}\left(c_{i j}+b_{j} u_{i}\right) z_{i j} \\
\text { Subject to } \quad \sum_{i \in I} z_{i j}=1 \quad \text { for } j \in J \\
\qquad z_{i j} \leq y_{i} \quad \text { for } i \in I, j \in J \\
y_{i}, z_{i j} \in\{0,1\} \quad \text { for } i \in I, j \in J
\end{array}
\end{aligned}
$$

Gradually we choose values of multipliers with purpose to maximize the lower bound. Usability of this technique is in general stated and proved in [7]. One of possible approaches to choosing the Lagrangean multipliers is, for example sub-gradient method. Other, more effective methods are described in publications [3], [9]. For the purpose of this article, sub-gradient method is sufficient; because it shows the possibility of solving the problem (1) - (5) for certain special input data.

Sub-gradient method is iterational method for subsequent modification of the values of multipliers vector $\boldsymbol{u}$ with the aim to maximize the lower bound of optimal solution of abovementioned problem. The procedure consists of solving the changed location problem by exact BBDual algorithm.

Let us label by $F\left(\boldsymbol{u}^{k}\right)$ the calculated value of objective function (6) for the $k$-th iteration of vector $\boldsymbol{u}$ labeled $\boldsymbol{u}^{k}$. Assuming that, the denoted by transition from vector $\boldsymbol{u}^{k}$ to the following $\boldsymbol{u}^{k+1}$ is realised in the direction of vector with an acute angle to the direction of greatest increase function $F$, i.e. in the direction of the gradient of the function near $\boldsymbol{u}^{k}$. Gradient of function $F$ labelled grad is a vector, whose components are partial derivatives of function $F(\boldsymbol{u})$ by all components of vector $\boldsymbol{u}$.

The algorithm of the method begins at initial not negative value of vector $\boldsymbol{u}^{0}$, chosen in advance, usually $\boldsymbol{u}^{0}$ $=\mathbf{0}$. Then, value of each new vector $\boldsymbol{u}$ is:

$\boldsymbol{u}^{k+1}=\max \left\{0, \boldsymbol{u}^{k}+\alpha \cdot\right.$ grad $\left./\|\operatorname{grad}\|\right\}$, where for $i \in I, k=$ $0,1,2, \ldots$

$\operatorname{grad}_{i}=\sum_{j \in J} b_{j} z_{i j}-a_{i} y_{i}$

Parameter $\alpha$ is chosen from interval $\left[\alpha_{\min }, \alpha_{\max }\right]$, where $\alpha_{\min }$ is a minimal value chosen in advance and $\alpha_{\max }=$ $\frac{H H-F\left(\boldsymbol{u}^{0}\right)}{\|\operatorname{grad}\|^{2}}$, where $H H$ is an upper bound of the value of the objective function (1) calculated for any feasible solution of problem (1) - (5).

Sub-gradient method finishes, when value of the objective function cannot grow any more or when change of its value is minimal, set in advance by defined value, certainty $\varepsilon$. 
The algorithm itself can be described as follows:

\section{- Input values:}

Choose $\boldsymbol{u}^{0}=\mathbf{0}, \alpha_{\min }, \varepsilon$.

- Algoritmus sub-gradient method:

1. Calculate $H H$, choose $k=0$

Solve the problem (6) - (9) using algorithm BBDual, calculate $F\left(\boldsymbol{u}^{k}\right)$;

2. Calculate $\operatorname{grad}_{i}=\sum_{j \in J} b_{j} z_{i j}-a_{i} y_{i}$, for $i \in I$, $\alpha_{\max }=\frac{H H-F\left(\boldsymbol{u}^{0}\right)}{\|\operatorname{grad}\|^{2}}$, choose $\alpha=2 \alpha_{\max } ;$

3. Calculate $\boldsymbol{u}^{k+1}$ using (10) and change the coefficients of the objective function (6). Solve the problem (6) (9) using algorithm BBDual, compute $F\left(\boldsymbol{u}^{k+1}\right)$;

4. If $F\left(\boldsymbol{u}^{k}\right) \geq F\left(\boldsymbol{u}^{k+1}\right)$, then $\alpha=\alpha / 2$

if $\alpha>\alpha_{\text {min }}$, then return to step 3, otherwise finish;

5. $k=k+1$

if $\left(F\left(\boldsymbol{u}^{k+1}\right)-F\left(\boldsymbol{u}^{k}\right)\right)>\varepsilon$, then return to step 2, otherwise finish.

\section{- Output values:}

The last calculated $F\left(\boldsymbol{u}^{k+1}\right)$ as a value of lower bound of the solution and $\boldsymbol{u}^{k+1}$ as a value of the multiplier vector.

Then, we have found out whether the optimization problem with the chosen facilities has a non-empty set of feasible solutions. To improve the quality of the final solution, obtained using Langragean relaxation, a heuristic method was designed, which removed or corrected infeasibility of the solution. When designing the method, an additional condition was stated to improve the compactness of groups of customers assigned to chosen facilities [3], [6].

The described technique shows that capacitated location problem can be solved for deterministic input data. In the following chapter possible fuzzy approach to the solution of abovementioned in vagueness conditions will be shown.

\subsection{Fuzzy approach in vague conditions}

The input capacities of the facilities $a_{i}$ for $i \in I$ are vague. They represent the serving ability of facilities for a period set in advance. This information is unknown in the time of designing the service system and in reality it has unstable varying values. The possibility of using fuzzy logic is shown in [5], [10], [11], [13].

Let us approximate the volumes of capacities $a_{i}, i \in I$ by a fuzzy set and describe it by a membership function, that represents the ratio in which a real value can be a part of this fuzzy set.

Let us introduce a fuzzy set of values approximately less than a crisp values $a$ labelled $\leq a$ and describe it by membership function $\mu_{\leq a}(x)$ for $x \in R$. Unknown, unsure real value will be approximated by the fuzzy set $\leq a$. Level of satisfaction in which a real value $x$ belong to fuzzy set $\leq a$, corresponds to a value of membership function $\mu_{\leq a}(x)$. It has values from interval $[0,1]$ like this:

$$
\begin{array}{ll}
\mu_{\leq a}(x)=1 & \text { for } x \leq a \\
\mu_{\leq a}(x)=\frac{a+p-x}{p} & \text { for } x \in(a, a+p) \\
\mu_{\leq a}(x)=0 & \text { for } x \geq a+p,
\end{array}
$$

and the graph of piecewise linear function $\mu_{\leq a}(x)$ is illustrated below :

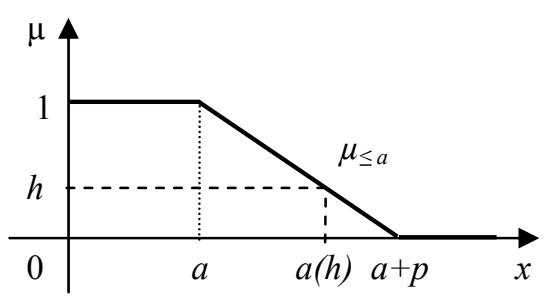

Fig. 1 Membership function of fuzzy set $\leq a$ $\left(\mu_{\leq a}(a(h))=h\right)$

For the problem $(1)-(5)$, let us assume, that we are able to determine approximately real service ability of facilities for certain time, represented by the capacities of individual facilities $a_{i}, i \in I$. To continue, let us assume, that we know approximate increases $p_{i}, i \in I$ of this volumes, when the service ability of these facilities, defined by capacities $a_{i}+p_{i}, i \in I$ is beyond the possibilities of the facilities. The values $a_{i}$ and $a_{i}+p_{i}, i \in I$ will be crisp values for introducing the fuzzy sets of values approximately less than crisp value $a_{i}$ with a toleration $p_{i}$ labelled $\leq a_{i}$ for every $i \in I$.

Level of belonging of a real value $a_{i}{ }^{*}$ into fuzzy set $\leq a_{i}$ is represented by a value of a membership function $\mu_{\leq a i}\left(a_{i}{ }^{*}\right)$ for $i \in I$ with values from interval $[0,1]$ and can be defined as:

$$
\begin{array}{ll}
\mu_{\leq a i}\left(a_{i}^{*}\right)=1 & \text { for } a_{i}^{*} \leq a_{i} \\
\mu_{\leq a i}\left(a_{i}^{*}\right)=\frac{a_{i}+p_{i}-a_{i}^{*}}{p_{i}} & \text { for } a_{i}^{*} \in\left(a_{i}, a_{i}+p_{i}\right) \\
\mu_{\leq a i}\left(a_{i}^{*}\right)=0 & \text { for } a_{i}^{*} \geq a_{i}+p_{i,}
\end{array}
$$

where the graph of the function of belonging is illustrated in Fig. 2.

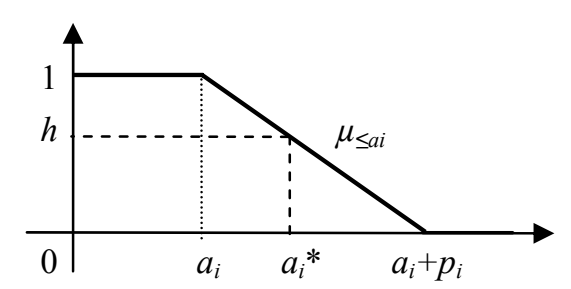

Fig. 2 Membership function of fuzzy set $\leq a_{i}$ $\left(\mu_{\leq a i}\left(a_{i}^{*}\right)=h\right)$

Then, we can define $\mu^{-1} \leq a i$ for $h \in[0,1]$ as:

$\mu^{-1} \leq a i(h)=h \cdot a_{i}+(1-h) \cdot\left(a_{i}+p_{i}\right), i \in I$ 
and they represent the biggest volumes of capacities $a_{i}(h)$ at the level of satisfaction $h$. The solution of problem (1)(5) for capacities at the level $h$ we will be indicated as the solution, that satisfies the capacity conditions (4) with the level of satisfaction over or equal $h$.

When the input data of the designed system is unsure, the value of the objective function is unsure, too. According to the minimization problem it will be approximated by a fuzzy set of "sufficiently small" values of the objective function. How we can obtain the crisp values that describe this fuzzy set?

The problem (1) - (5) will be solved with chosen crisp values of the capacities $a_{i}$ and $a_{i}+p_{i}, i \in I$. We obtain solutions labelled $(\boldsymbol{y}, \boldsymbol{z})$ and $\left(\boldsymbol{y}^{p}, \boldsymbol{z}^{p}\right)$. The computed values of the objective functions will be labelled $F_{\min }=F\left(\boldsymbol{y}^{p}, \boldsymbol{z}^{p}\right)$ and $F_{\max }=F(\boldsymbol{y}, \boldsymbol{z})$ according to the fact, that when the volumes of capacities are higher $a_{i}+p_{i}$ the objective function obtains lower value than when the capacities are $a_{i}, i \in I$.

Let us denote by the value $F^{*}$ of the objective function $F\left(y^{*}, z^{*}\right)$, where $\left(y^{*}, z^{*}\right)$ is the solution of problem (1) (5) for the capacities $a_{i}{ }^{*}, i \in I$. The unknown value $F^{*}$ belong to the fuzzy set of „sufficiently small“ values of the objective function labelled $\geq F$ with the level of satisfaction defined by the value of the membership function $\mu_{\geq F}\left(F^{*}\right)$ defined as:

$\mu_{\geq F}\left(F^{*}\right)=1 \quad$ for $F^{*} \leq F_{\min }$

$\mu_{\geq F}\left(F^{*}\right)=\frac{F_{\text {max }}-F^{*}}{F_{\text {max }}-F_{\text {min }}}$ for $F^{*} \in\left(F_{\min }, F_{\max }\right)$

$\mu_{\geq F}\left(F^{*}\right)=0 \quad$ for $F^{*} \geq F_{\max }$,

where the graph of the function is illustrated in Fig. 3. Then, we can define $\mu^{-1} \geq F$ for $h \in[0,1]$ as:

$\mu^{-1} \geq F(h)=h \cdot F_{\min }+(1-h) \cdot F_{\max }$

and they represent the value of the objective function when the capacities are $a_{i}(h)=h \cdot a_{i}+(1-h) \cdot\left(a_{i}+p_{i}\right), i \in I$ on the level of satisfaction $h$.

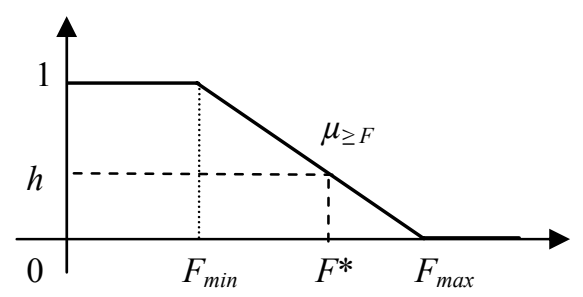

Fig. 3 Membership function of fuzzy set $\geq F$ $\left(\mu_{\geq F}\left(F^{*}\right)=h\right)$

The aim is to find the vector of variables $\left(y^{*}, z^{*}\right)$ as solution of problem (1) - (5) to get the lowest possible value of the objective function with the highest possible level of satisfaction and the capacity conditions satisfied.

With this aim, we transform the problem of linear programming problem (1) - (5) with model:

Maximize $h$

Subject to $F(\boldsymbol{y}, \boldsymbol{z}) \leq h \cdot F_{\min }+(1-h) \cdot F_{\max }$

ISSN $1335-8243$ (print) (C) 2011 FEI TUKE www.aei.tuke.sk

$$
\begin{aligned}
& \sum_{j \in J} b_{j} z_{i j} \leq h . a_{i}+(1-h) .\left(a_{i}+p_{i}\right), i \in I \\
& (2),(3),(5)
\end{aligned}
$$

with the purpose to find acceptable $h^{*}$, that maximizes the level of satisfaction of the original solution of problem (1) - (5). The expression $h \cdot F_{\min }+(1-h) \cdot F_{\max }$ is, however nonlinear in variables $\boldsymbol{y}, \boldsymbol{z}$ and $h$, but linear for defined, selected $h$. On this principle, the iterational method Tanaka - Asai [12] is based. It uses the way of solving the linear problem with model:

\section{Minimize $\quad F(\boldsymbol{y}, \boldsymbol{z})$}

$$
\begin{array}{ll}
\text { Subject to } \quad F(\boldsymbol{y}, \boldsymbol{z}) & \leq h \cdot F_{\min }+(1-h) \cdot F_{\max } \\
& \sum_{j \in J} b_{j} z_{i j} \leq h \cdot a_{i}+(1-h) \cdot\left(a_{i}+p_{i}\right), i \in I \\
& (2),(3),(5)
\end{array}
$$

for chosen $h_{0}$, continually increasing $h$ by given $\Delta h>0$. If a feasible solution exists on the level $h$, then we repeat the solution of problem with $h$ increased by $\Delta h$. In other case, the iteration process finishes. The level $h^{*}$ and the level of satisfaction of the last step solution is also the definitive solution of the described technique.

Using the described method, it is possible to find the acceptable level of satisfaction of the solution of problem (1) - (5), but it does not guarantee that it will be an optimal value as well. The problem is, how to choose the suitable value of $h_{0}$ and $\Delta h$. For example, for $h_{0}=0.1$ and $\Delta h=0.01$ we get an acceptable solution for all $h<0.48$. Thus, $h^{*}=0.47$. If we choose $h_{0}=0.1$ and $\Delta h=0.05$, then we can get an unacceptable solution when $h=0.55$. The level of satisfaction will be $h^{*}=0.5$ and it is a higher value than $h^{*}=0.47$.

In this article we will use another possible application of Tanaka - Asai method. It is a heuristic method, based on solving the crisp problem (1) - (5) with continually changing the capacities.

For $h$ chosen in advance from interval $[0,1]$ the capacities are $a_{i}(h)=h \cdot a_{i}+(1-h) \cdot\left(a_{i}+p_{i}\right), \quad i \in I$. The solution of problem is a vector $\left(y^{h}, z^{h}\right)$ and the value of the objective function labelled $F_{h}=F\left(y^{h}, z^{h}\right)$ depending on the input value $h$. By using the formula (14) we calculate $\mu_{\geq F}\left(F_{h}\right)$ as a level of satisfaction, in which the calculated value $F_{h}$ belong to the fuzzy set of "sufficiently small" values.

Every solution of problem may be acceptable or unacceptable depending on satisfying capacity conditions (4) according to the chosen input capacities $a_{i}(h), i \in I$. The minimal demanded service ability of the $i$-th facility is represented by a total $\sum_{j \in J} b_{j} z_{i j}$. At the same time, the condition $\sum_{j \in J} b_{j} z_{i j} \leq a_{i}(h)$ ensures that the facility has a sufficient volume of capacities, that it can cover the requests of the assigned customers.

The solution of problem is unacceptable, if some of the chosen facilities cannot satisfy requirements of assigned customers, which means that for some $i \in I_{\text {selection, }}$ $\sum_{j \in J} b_{j} z_{i j}>a_{i}(h) . I_{\text {selection }} \subseteq I$ was defined as a set of chosen 
facilities in the stated solving of problem. If we label $a_{i}{ }^{*}$ $=\sum_{j \in J} b_{j} z_{i j}$, then $a_{i}^{*}$ is the capacity of the $i$-th facility and it belong to the fuzzy set $\leq a_{i}$ with the level of satisfaction defined by the value of the function $\mu_{\leq a i}\left(a_{i}{ }^{*}\right)$ according to (13).

What will be the level of satisfaction of satisfying the capacity condition of the obtained solution?

If the $i$-th facility does not satisfy the capacity requirement, than $a_{i}{ }^{*}>a_{i}(h)$ and $\mu_{\leq a i}\left(a_{i}^{*}\right)<h$, which is illustrated in Figure 4. From the values $\mu_{\leq a i}\left(a_{i}{ }^{*}\right)$ calculated for all these facilities, we choose the lowest and it will determine the level of satisfaction of satisfying the capacity conditions. Let us label it $h^{*}$. In the case, that the solution of problem reaches a level of unacceptability so high, that the capacity volume $a_{i}^{*}>a_{i}+p_{i}$, we do not accept the solution, because the facility with zero level of satisfaction was located. For the facilities, that satisfy the capacity condition $a_{i}^{*} \leq a_{i}(h)$, it is $\mu_{\leq a i}\left(a_{i}{ }^{*}\right) \geq h$. In this case the level of satisfaction $h^{*}=h$.

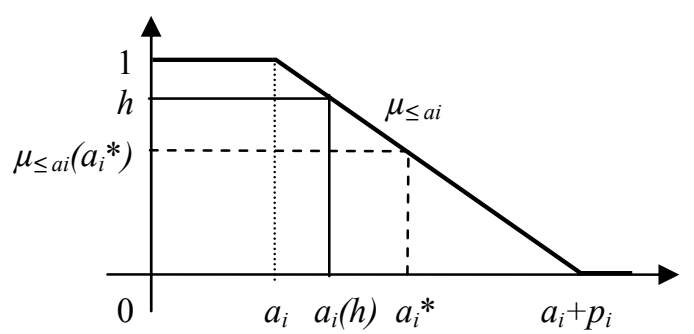

Fig. 4 Membership function of fuzzy set $\leq a_{i}$

Thus, $h^{*}$ will be the level of satisfaction of solving the problem $\left(y^{h}, z^{h}\right)$ with the value of the objective function $F_{h}=F\left(y^{h}, z^{h}\right)$, that belong to the fuzzy set of "sufficiently small" values with the level of satisfaction $\mu_{\geq F}\left(F_{h}\right)$ assuming that $h^{*} \leq \mu_{\geq F}\left(F_{h}\right)$. In other case, we do not accept the solution, because although we reached a high level of satisfying the capacity conditions, but the financial costs is also uncceptably high.

The described technique will be repeated by solving the crisp problem (1) - (5) with new capacities defined by the values of inverse membership function according to:

$\mu^{-1} \leq a i\left(h^{*}\right)=h^{*} \cdot a_{i}+\left(1-h^{*}\right) \cdot\left(a_{i}+p_{i}\right), i \in I$.

The suggested heuristic method using the level of infeasibility of solution of problem (1) - (5) in accordance with the chosen level $h$ is a problem to find an optimal level of satisfaction $H$ for the final solution of problem, which satisfies the capacity conditions with highest possible level, when the value of the objective function is "sufficiently small". Description of the suggested algorithm:

\section{- Input values:}

Set crisp values $a_{i}$ and $a_{i}+p_{i}$ for $i \in I$, we choose a step $\Delta h>0$.

- Algorithm using the level of unacceptability:

0 . $h=0$ and intermediate variable $h h=0$.

1. Solve the problem (1) - (5) for capacities $a_{i}, i \in I$, we ISSN 1335-8243 (print) C 2011 FEI TUKE www.aei.tuke.sk get $(\boldsymbol{y}, \boldsymbol{z})$ a $F_{\max }$.

Solve the problem (1) - (5) for capacities $a_{i}+p_{i}, i \in I$, we get $\left(\boldsymbol{y}^{p}, \boldsymbol{z}^{p}\right)$ a $F_{\text {min }}$.

2. Put $h=h+\Delta h$. If $h \geq 1$, then finish, otherwise $h h=$ $h$.

3. Calculate $a_{i}(h)=h \cdot a_{i}+(1-h) \cdot\left(a_{i}+p_{i}\right)$ for $i \in I$.

4. Solve the problem (1) - (5) for capacities $a_{i}(h), i \in I$, we get $\left(\boldsymbol{y}^{h}, \boldsymbol{z}^{h}\right)$ a $F\left(\boldsymbol{y}^{h}, \boldsymbol{z}^{h}\right)=F_{h}$ and a set $I_{\text {selection. }}$.

Calculate $\mu_{\geq F}\left(F_{h}\right)$ according to (14) and

$\mu_{\leq a i}\left(\sum_{j \in J} b_{j} z_{i j}\right)$ according to (11) for $i \in I_{\text {selection }}$.

5. If there exists $i \in I_{\text {selection }}$ with $\sum_{j \in J} b_{j} z_{i j} \geq a_{i}+p_{i}$, then go to step 2 with $h=h h$,

otherwise, if $\sum_{j \in J} b_{j} z_{i j} \leq a_{i}(h)$ for all $i \in I_{\text {selection }}$ then $h^{*}=h$ and go to step $\mathbf{6}$, otherwise

$h^{*}=\min \left\{\mu_{\leq a i}\left(\sum_{j \in J} b_{j} z_{i j}\right): \sum_{j \in J} b_{j} z_{i j}>a_{i}(h), i \in I_{\text {selection }}\right\}$.

6. If $h^{*} \leq \mu_{\geq F}\left(F_{h}\right)$, then include $h^{*}$ into a set $\left\{h^{*}\right\}$.

7. Put $h=h^{*}$ and go to step 3 .

- Output values:

The optimal level of satisfaction $H=\max \left\{h^{*}\right\}$.

\section{NUMERICAL EXPERIMENTS}

To test the approach, the associated algorithms were implemented using Delphi 7 programming environment. To perform the numerical experiments, Pentium $4 / 3 \mathrm{GHz}$ computer with $1 \mathrm{~GB}$ of RAM was used. The approach to the capacitated facility location problem was tested with data originating at Slovak road network with 2907 dwelling places, where each of them represents one customer. 71 centres of the former districts form the set of the possible facility locations.

We tested the described algorithm on chosen problems with operating costs $c_{i j}$, that were modelled by the formula $c_{i j}=\left(e_{0} d_{s i}+e_{1} d_{i j}+g_{i}\right) . b_{j}$, for $i=1, \ldots, 71$ and $j=1, \ldots$, 2907, where :

$d_{s i}$ is the distance of the primary source $s$ from the facility in the location $i$,

$d_{i j} \quad$ is a distance of the $i$-th facility from customer $j$,

$e_{0} \quad$ are unit costs to obtain the facility $i$ with a primary source $s$,

$e_{1}$ are unit costs to obtain a customer $j$ with facility in the location $i$

$g_{i}$ are several added overhead costs for operating the facility $i$,

$b_{j} \quad$ are the demands of $j$-th customer.

We choose three various values for the input values of fixed costs $f_{i}$, further three various unit costs $e_{1}$ and one chosen primary source in the place $s$ that will ensure each selected facilities.

For data generated in this way, 9 problems labelled f-e were created, where the numbers $\mathrm{f}=3,5,7$ represent individual input vectors $f_{i}$ under established labelling of www.versita.com/aei 
files with input data. In similar way the number e $=2,4,6$ represents unit costs $e_{1}$. The input capacities of facilities $a_{i}, i=1, \ldots, 71$ were generated in a way, that for all facilities have equal values. Each problem was solved for chosen $\Delta h=0,1$ and crisp values $p_{i}=a_{i}, i=1, \ldots, 71$ by Lagrangean relaxation of the capacity conditions using sub-gradient method to choose the Langragean multipliers properly.

Individual solutions of each problem for selected capacities are described in the graphs and tables with solutions, we got when testing the described algorithm.

Considering the quality of the solutions is related to finding the optimal level of satisfaction of the solution $H$ depending on satisfying the capacity conditions defined by the value of the membership function $\mu_{\leq a}$ of fuzzy interval $\leq a$ and the value $\mu_{\geq F}$, that represents the level of satisfaction of "sufficiently small" value of the objective function.

Table 1 Output values of problem 3-2

\begin{tabular}{|c|c|c|}
\hline$h$ & $\mu_{\leq a}$ & $\boldsymbol{\mu}_{\geq \boldsymbol{F}}$ \\
\hline 0 & 0 & 1 \\
\hline 0.1 & 0.1 & 0.68714 \\
\hline 0,2 & & \\
\hline 0,3 & & \\
\hline 0.4 & 0.20855 & 0.79021 \\
\hline 0.20855 & & \\
\hline 0.5 & 0.30688 & 0.35190 \\
\hline 0.30688 & 0.20855 & 0.77512 \\
\hline 0.6 & 0.301487 & 0.34774 \\
\hline 0.30149 & & \\
\hline 0.7 & 0.37825 & 0.13170 \\
\hline 0.37825 & 0.20855 & 0.79854 \\
\hline 0.8 & 0.53550 & 0.25403 \\
\hline 0.53550 & 0.28476 & 0.35346 \\
\hline 0.28476 & & \\
\hline 0.9 & 0.62862 & 0.05414 \\
\hline 0.62862 & 0.27974 & 0.35138 \\
\hline 0.27974 & & \\
\hline 1 & 0.55688 & 0 \\
\hline 0.55688 & 0.27974 & 0.35294 \\
\hline
\end{tabular}

In Table 1 we present the solutions of problem 3-2 with given values of the functions $\mu_{\leq a}$ (second column) and $\mu_{\geq F}$ (third column). The rows in table represent gradual solving of problem (1) - (5) for the capacities depending on the chosen level $h$ (first column) using the suggested algorithm. Blank rows are unacceptable solutions, where the volume of the demands of the customers assigned to some facility exceeded the limit of capacities $a+p$.

We excluded these solutions from Table 1 and sorted individual partial solutions ascending by the values $h \in[0$, 1]. New Table 2 then offers a new point of view at choosing these values using the described algorithm. Each row is an acceptable final solution of the crisp problem with capacities $a_{i}=h \cdot a_{i}+(1-h) .\left(a_{i}+p_{i}\right)$ corresponding to the value $h$ in the first column, where for $h=0$ those are the highest values $a_{i}+p_{i}, i=1, \ldots, 71$ and for $h=1$ values $a_{i}$, $i=1, \ldots, 71$.

We tried to illustrate the use of the suggested algorithm when solving chosen problems graphically as well. The output values from Table 2 are shown on Graph 1 using linear interpolation.

Table 2 Sorted output values of problem 3-2, optimal $H=0.30688$

\begin{tabular}{|c|c|c|}
\hline $\boldsymbol{h}$ & $\boldsymbol{\mu}_{\leq \boldsymbol{a}}$ & $\boldsymbol{\mu}_{\geq \boldsymbol{F}}$ \\
\hline 0 & 0 & 1 \\
\hline 0.1 & 0.1 & 0.68714 \\
\hline 0.30688 & 0.20855 & 0.77512 \\
\hline 0.37825 & 0.20855 & 0.79854 \\
\hline 0.4 & 0.20855 & 0.79021 \\
\hline 0.5 & $\mathbf{0 . 3 0 6 8 8}$ & 0.35190 \\
\hline 0.53550 & 0.28476 & 0.35346 \\
\hline 0.55688 & 0.27974 & 0.35294 \\
\hline 0.6 & 0.30149 & 0.34773 \\
\hline 0.62862 & 0.27974 & 0.35138 \\
\hline 0.7 & 0.37825 & 0.13170 \\
\hline 0.8 & 0.53550 & 0.25403 \\
\hline 0.9 & 0.62862 & 0.05414 \\
\hline 1 & 0.55688 & 0 \\
\hline
\end{tabular}

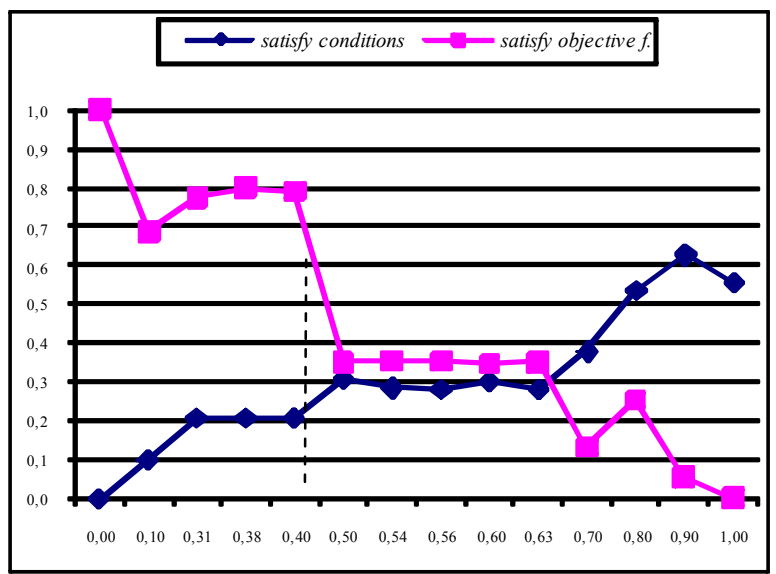

Fig. 5 The graph of partial solutions of problem 2-3

Horizontal axis in the Fig. 5 represents the values $h$ and the vertical one corresponding values of the membership functions $\mu_{\leq a}$ as rhombuses on one curve and $\mu_{\geq F}$ as rectangles on another curve depending on the capacities $a_{i}(h)$ for $i \in I$. Corresponding values of the graph are stated in Table 2.

The curves from Fig. 5 are in fact nonlinear. It is related to heuristic method of solution of the crisp problem $(1)-(5)$. There is a new rearrangement of the distribution system suggestion. It is always a new selection of facilities with a new selection of customers assigned to these facilities and with other value of the objective function. 
Values on the curves approach the point, where they meet and their intersect changes. At first the level of satisfaction of satisfying the capacity conditions rises and the level of satisfaction of the value of the objective function declines and then vice versa. Fig. 6 represents solution of problem 7-6 and it shows, that several irregularities can occur. Graphically, they are represented by the changes of the line graphs.

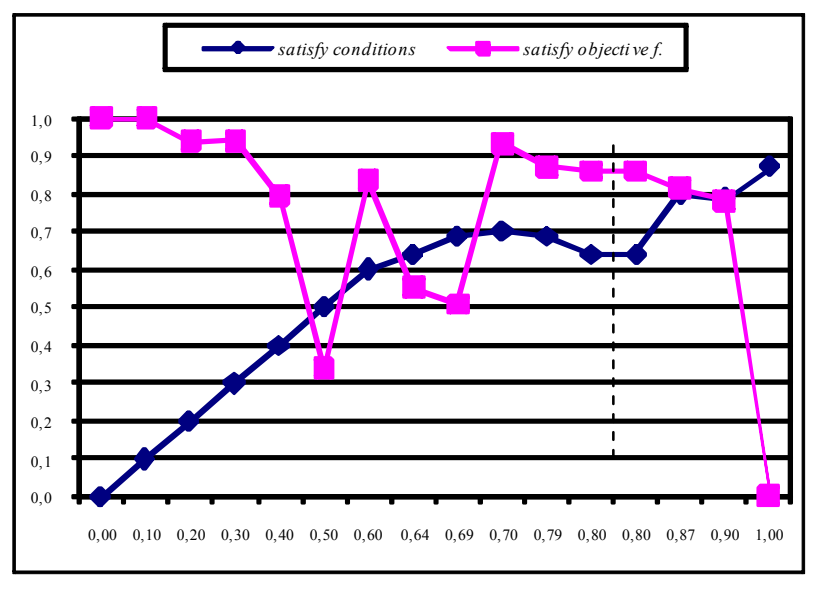

Fig. 6 The graph of partial solutions of problem 7-6

The optimal value $H$ is marked on the graphs by a dashed line as a maximum of the set $\left\{h^{*}\right\}$, which values are graphically represented by rhombuses situated right under relevant rectangles. The solution of problem 7-6 reaches the highest possible level of satisfaction $H=0.801$ when the capacities is $a_{i}=h \cdot a_{i}+(1-h) .\left(a_{i}+p_{i}\right), i \in I$ for $h=0.87$.

Table 3 Output values of the 9 solutions

\begin{tabular}{|c|c|c|c|}
\hline problem & $\boldsymbol{h}$ & $\boldsymbol{H}$ & $\boldsymbol{\mu}_{\geq \boldsymbol{F}}$ \\
\hline $3-2$ & 0,5 & 0,307 & 0,352 \\
\hline $3-4$ & 0,5 & 0,432 & 0,615 \\
\hline $3-6$ & 0,537 & 0,509 & 0,524 \\
\hline $5-2$ & 0,671 & 0,342 & 0,629 \\
\hline $5-4$ & 0,6 & 0,429 & 0,722 \\
\hline $5-6$ & 0,6 & 0,537 & 0,697 \\
\hline $7-2$ & 0,877 & 0,848 & 1 \\
\hline $7-4$ & 0,814 & 0,656 & 0,997 \\
\hline $7-6$ & 0,870 & 0,801 & 0,816 \\
\hline
\end{tabular}

Table 3 gives information about the final solution of 9 chosen problems. The first column is the title of problem depending on input values of fixed costs (first number) and unit costs (second number). The third column shows, how the reached values of the objective function (1) belong to the fuzzy set of "sufficiently small" values of the objective function. Fourth column in table represents the level of satisfying the capacity conditions (4) of the final solution depending on the input quantities $h$ written in the second column.

From Table 3 we can see, that output gives information about the fact, that each of the 9 problems reached an optimal level $H$ for various input values of expense ratios using the implemented algorithm.

Calculation time of the whole problem is determined by the number of solutions of the crisp problem (1) - (5), where one problem solved by the Langragean relaxation of the capacity conditions with use of the sub-gradient method to choose Langragean multipliers properly takes on average $2.2 \mathrm{~min}$.

\section{CONCLUSIONS}

The suggested technique for solving capacitated location problem with uncertain capacity limitations using the theory of fuzzy sets gives results shown in Table 3 for all 9 chosen problems. If there were an exact method for solving the crisp problem (1)-(5), several changes in graph of illustrated curves would not occur. The changes are caused by the fact, that the problem was solved by the heuristic method described in sub-chapter 2.2.

The quality of the obtained solution can be improved by a finer division of the fuzzy intervals $\left(a_{i}, a_{i}+p_{i}\right), i \in I$, i.e. by choosing for example a lower value $\Delta h$. Another option is to remove or at least improve unacceptability of partial solutions of the crisp problem (1) - (5).

In this paper we developed and tested possible fuzzy approach to the capacitated facility location problem. Results of the performed experiments have arisen several new questions, which could be worth to follow in a possible future research. It can find applications in various fields of logistics with various conditions of uncertainty of considered input objects.

\section{ACKNOWLEDGEMENT}

This work has been supported by research project grant 4/2007 of the Faculty of Management and Informatics at University of Žilina and by research project VEGA 1/0361/10.

\section{REFERENCES}

[1] ERLENKOTTER, D.: A Dual-Based Procedure for Uncapacitated Facility Location. Operations Research, Vol. 26, No. 6, 1978, pp. 992-1009.

[2] DREZNER, Z.: Facility Location Application and Theory. Springer Verlag, Berlin, Heidlberg, NewYork, 2004, 457 p.

[3] GABRIŠOVÁ, L.: Optimalization of the Location of the Control and Service Facilities in Systems in Vague Conditions. PhD thesis, Faculty of Management Science and Informatics, University of Žilina, 2008, 94 p.

[4] JANÁČEK, J.: Service System Design in the Public and Private Sectors. In: Proceeding of the Quantitative Methods in Economics, Virt, 2004, pp. 101-108.

[5] JANÁČEK, J.: Fuzzy Approach to the Capacitated Location Problem. In: Proceedings of the conference "Operations Research", Society for Operations Research, Bremen, German, 2005. 
[6] JANÁČEK, J.: Customers' pool partitioning to compact sub-areas. In: Advances in Transport Systems Telematics, Warszawa, 2008, pp. 117-124.

[7] JANÁČEK, J. - BUZNA, L': Facility Location in Distribution Systems. EDIS, University of Žilina, 2007, 142 p.

[8] JANÁČEK, J. - BUZNA, L': An acceleration of Erlenkotter-Körkel's algoriths for uncapacitated facility location problem. In: Annals of Operations Research, Vol. 164, 2008, pp. 97-109.

[9] JANÁČEK, J. - GÁBRIŠOVÁ, L.: Lagrangean Relaxation Based Approximate Approach to the Capacitated Location Problem. Communications: Scientific Letters of the University of Žilina, Vol. 8, No. 3, 2006, pp. 19-24.

[10]NOVÁK, V.: Fuzzy Sets and Their Applications. (in Czech) Praha, 1986, 278 p.

[11]RAMÍK, J. - VLACH, M.: Generalized Concavity in Fuzzy Optimization and Decision analysis. Kluwer Academic Publishers, Boston, 2002, 296 p.
[12] TANAKA, H. - ASAI, K.: Fuzzy Linear Programming Problems with Fuzzy Numbers. Fuzzy Sets and System, 13, (1984)1-10.

[13] TEODOROVIČ, D. - VUKADINOVIČ, K.: Traffic Control and Transport Planning: A Fuzzy Sets and Neural Networks Approach. Kluver Academic Publishers, Boston, Dordrecht, London, 1998, 387 p.

Received September 2, 2010, accepted January 28, 2011

\section{BIOGRAPHY}

Lýdia Gábrišová was born on 19.8.1966. In 1989 she graduated (Mgr.) with distinction at the department of Approximate and Numerical Method of the Faculty of Mathematics, Physics and Informatics at Comenius University of Bratislava. She gains PhD. in „Applied informatics" in 2008; her thesis title was "Optimalization of the Location of the Control and Service Facilities in Systems in Vague Conditions". Since 2001 she is working as Assist. Prof. in the Department of Mathematical Methods of the Faculty of Management and Informatics at University of Žilina. 